

\title{
The SuperB Detector
}

\author{
Nicolas ARNAUD*i \\ Laboratoire de l'Accélérateur Linéaire, CNRS/IN2P3 et Université Paris-Sud-Orsay, France \\ E-mail: narnaudelal.in2p3.fr
}

The SuperB experiment is a next generation Super Flavour Factory expected to accumulate $75 \mathrm{ab}^{-1}$ of data at the $\Upsilon(4 S)$ in five years of nominal running. In addition, SuperB will be able to run from the $\psi(3770)$ up to the $\Upsilon(6 S)$, while a polarized electron beam enables unique physics opportunities. In this article, the detector designed for this new facility, to be built on the campus of the Tor Vergata University (near Roma) by the "Nicola Cabibbo" laboratory, is discussed.

XXIst International Europhysics Conference on High Energy Physics

21-27 July 2011

Grenoble, Rhône-Alpes France

\footnotetext{
*Speaker.

†n behalf of the SuperB detector collaboration.
} 


\section{Introduction}

SuperB is a new generation flavour factory which will be built on the campus of the Tor Vergata University near Roma. This international project lead by the INFN is rated first in the list of 14 flagship projects which have been selected in the new national research plan approved by the Italian government in December 2010. The "Nicola Cabibbo Laboratory", the entity which will build and operate the SuperB infrastructure, has been established in September 2011.The SuperB physics program $[1,2,3]$ is very ambitious: to unravel the flavour structure of the New Physics by probing many observables in a wide range of decays. In addition, SuperB will perform precision tests of the Standard Model thanks to its huge dataset: at least $75 \mathrm{ab}^{-1}$ at the $\Upsilon(4 S)$ resonance, accumulated in 5 years of running at an instantaneous luminosity of $10^{36} \mathrm{~cm}^{-2} \mathrm{~s}^{-1}$. The performances of the SuperB collider [1, 4] are due to an innovative design of the interaction region [5] successfully tested at DAФNE [6]. SuperB will also benefit from unique features of the machine, namely an electron beam polarized at $\approx 80 \%$ and a wide range of running energies: from the $\psi(3770)$ to the $\Upsilon(6 S)$. The SuperB approach to the physics is complementary to the other flavour experiments operating in the coming decade, in particular $\mathrm{LHCb}$ and its future upgrade.

\section{The SuperB detector}

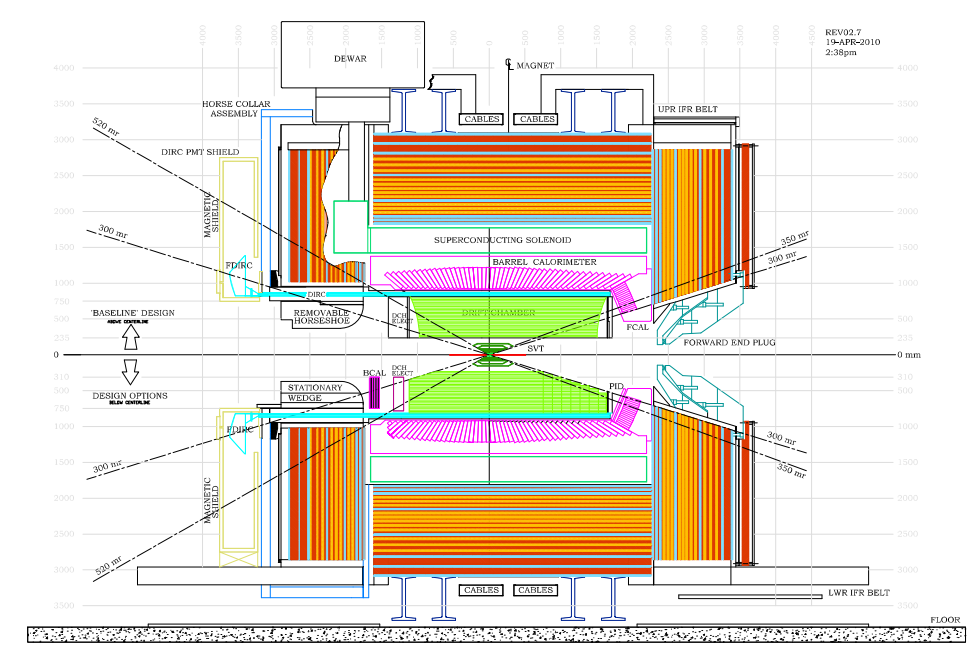

Figure 1: Side view of the SuperB detector. The upper half shows the baseline concept while the bottom half adds a number of detector options, among which a backward calorimeter and a forward PID detector.

The SuperB detector [7] is based on BABAR [8] and will reuse some of its components: the coil, the quartz bars of the DIRC [9], the barrel electromagnetic calorimeter and part of the structure. The detector will cost an extra $50 \mathrm{M} €$ which will be covered by the INFN (for about 50\%) and by the international partners of the project: Canada, France, Germany, Poland, Russia, Spain, UK and USA. Requests to the corresponding funding agencies are in progress while Memoranda Of Understanding are being drafted. Figure 1 shows a side view of the SuperB detector : from the inside to the outside, its differents components are the following.

- A tracking system made of a 6-layer vertex detector (SVT) and a 40-layer drift chamber. Charged tracks are bent by a $1.5 \mathrm{~T}$ magnetic field provided by a superconducting coil. 
- A ring-imaging Cherenkov detector, the "Focusing DIRC" (FDIRC), which is the main device for charged particle identification (PID) [10] in the SuperB barrel region.

- An electromagnetic calorimeter (EMC) covering both the barrel and forward regions.

- An instrumented flux return (IFR) used to detect muons and neutral hadrons.

- The Electronics, Trigger and Data Acquisition system covering all online SuperB activities.

Active R\&D activities continue in many areas to improve and optimize the SuperB design; they will culminate in the publication of the detector TDR in 2012 - contemporaneously with the machine and physics TDRs. Despite significant changes in the running conditions w.r.t. BABAR (collider less asymmetric, luminosity increased by two orders of magnitude, higher background), the SuperB performances will be better. First, a new SVT layer will be added at a radius of $1.5 \mathrm{~cm}$ to compensate the reduced boost: $\beta \gamma=0.24$ instead of 0.56 . Then, new photon cameras $(\times 25$ smaller in volume) have been designed for the FDIRC to compensate for the background increase. Moreover, many devices are completely new and benefit from technological advances and the experience of the LHC experiments: the SVT layers 1 to 5, the DCH, the forward EMC, the IFR detectors and absorber layout and finally all the SuperB electronics. The trigger is designed for a first level (hardware) output rate of $150 \mathrm{kHz}$ (50\% headroom) and a minimal deadtime. The logging rate after the high-level trigger should be $\approx 2$ Gbyte/s. Finally, two additional detectors are being developed to improve the SuperB hermiticity: a backward calorimeter and a forward PID detector. Their technologies have been selected and room reserved in the SuperB layout. Prototypes will be assembled and tested in the coming months; if they are successful, these two options will be included in the baseline. All the current works are based on many simulation studies, done with either a fast parametric framework ("FastSim") or a full Geant4-based simulation code ("Bruno").

\section{Conclusions}

SuperB is a ambitious project which will start taking data in Italy in 2017. Many aspects of both the machine and the detector are very challenging - such as the physics program itself. International collaborations are being formed and there are still many opportunities to join them.

\section{References}

[1] M. Bona et al., SuperB Conceptual Design Report, arXiv: 0709.0451 (2007).

[2] B.O'Leary et al., The SuperB Physics Progress Report, arXiv: 1008.1541 (2010).

[3] B. Meadows et al., The impact of SuperB on flavour physics, arXiv:1109.5028 (2011).

[4] M. Biagini et al., The SuperB Progress Report, arXiv:1009.6178 (2010).

[5] P. Raimondi in 2nd LNF Workshop on SuperB, Frascati, Italy (2006) and in Proceedings of Particle Accelerator Conference (PAC 07), Albuquerque, USA, 32 (2007).

[6] M. Zobov et al., Phys.Rev.Lett. 104174801 (2010).

[7] E. Grauges et al., The SuperB Detector Progress Report, arXiv: 1007.4241 (2010).

[8] B. Aubert et al., (the BABAR Collaboration), Nuclear Instruments and Methods A 479 (2002) 1-116.

[9] I. Adam et al., Nuclear Instruments and Methods A 538, 281 (2005).

[10] N. Arnaud, "Charged particle identification (PID) for SuperB" in these proceedings. 